\title{
Scheduling Model and Algorithm for Collaborative Product Design Based on MD
}

\author{
Xiaolei Wang ${ }^{1, a^{*}}$, Meiliang $\mathrm{He}^{2, \mathrm{~b}}$ and Quan $\mathrm{Xu}^{1, \mathrm{c}}$ \\ ${ }^{1 *}$ Department of Mechanical Engineering, North China Electric Power University, Baoding, hebei, \\ China \\ ${ }^{2}$ Department of Economics and Trade, Hebei Finance University, Baoding, hebei, China

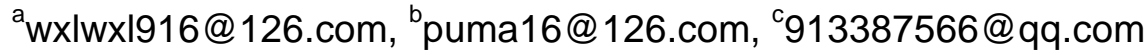

Keywords: Collaborative product design, Scheduling model, Matching degree, Genetic algorithm Abstract. In order to improve the efficiency of collaborative product design, project task and team should be allocated and scheduled reasonably. Firstly, the matching degree between task and team was defined. Then, trading-off project duration and cost, multi-objective scheduling model of collaborative product design based on matching degree was established, and the improved genetic algorithm was redesigned to solve the scheduling model. Through an example of collaborative design, the feasibility and validity of the proposed model and algorithm were verified. Simulation result shows that the algorithm has advantages in precision and convergence speed.

\section{Introduction}

With the rapid development of technology and the increase of product complexity, the division of labor is more and more specialized, so that the core enterprise needs to joint with customer, supplier and research institute to collaboratively work. Through cross-organizational collaborative product design (CPD), it can realize the maximization of resource integration and the improvement of design efficiency. However, in the process of collaborative product design, the diversity of design agent, and interdependence and mutual restriction between tasks, they make collaborative innovative design process become more complicated. Therefore, design task and resource should be reasonably allocated and scheduled to shorten the development cycle and reduce cost.

About task and resource allocation of collaborative design, some researches focused on task identification, analysis of task relationships and task scheduling based on Petri net[1] and design structure matrix (DSM)[2]. Some of other researches focused on establishing the scheduling optimization model for collaborative design and model solution algorithms[3,4,5,6]. In addition, currently, in the study of capability matching, Wu \& Qin proposed a resource capability measuring method and resource capability deployment mechanism by mapping RTCI to RPCI[7]. Zhang \& Li simulated collaborative product development process based agent, design agent selected his partner according to the ability and character matching degree[8].

In the process of CPD, design tasks are accomplished by different teams. The previous researches have focused on the matching between tasks and team members based on technical capability or designer preference. However, taking design team as a whole, from macro perspective, how to realize task-team reasonable matching? For design partner selection or task assignment, they need measure team comprehensive capacity.

Motivated by the above observations, firstly, competency elements of collaborative product design are presented. Then, matching degree between task and team is defined and its computing model is constructed. Furthermore, multi-objective scheduling model for collaborative product design based on matching degree is established, and the improved genetic algorithm is designed to solve the scheduling mode. Finally, an example is solved successfully to illustrate the feasibility and validity of the proposed model and algorithm. 


\section{Multi-Objective Scheduling Model of CPD}

Competency elements of collaborative product design. For cooperative task allocation, it is necessary to analyze the required capability for design task. It not only includes technical level, innovation capability, but also includes software and hardware resources and social resource[9]. The competency elements for CPD are listed in Table 1.

Table 1 Competency elements of collaborative product design

\begin{tabular}{lll}
\hline Goal & high efficiency and high quality \\
\hline Core competency & $\begin{array}{l}\text { team innovation capability } \\
\text { team communication capability }\end{array}$ & team learning capability \\
Basic & team executive capability & team service consciousness \\
competency & team technical capability & team management capability \\
Basic resource & information resource & hardware and software resource \\
& brand resource & social net resource
\end{tabular}

Definition of matching degree. Matching degree(MD) is a concept to measure the fitness between elements. As an example, when matching project task with technical team, if the matching degree is too low, it manifests that technical team's capabilities and resources are not enough to support them to complete the task. Higher matching degree ensures that team can accomplish the task high-efficiency and high-quality. But if matching degree is too high, it means cost rise. Thus, matching degree between task-team should be kept in a reasonable range.

The task-team matching degree model is constructed in two ways, one is personnel capability of technical team, and another is the available resources. The matching degree $T C_{i j}$ between task $i$ and team $j$ at the dimension of personnel capabilities is defined as follows:

$$
T C_{i j}=\sum_{p=1}^{8} \alpha_{i}^{p}\left(1 \pm \sqrt{\frac{\left(g_{i j}^{p}\right)^{2}-\left(e_{i}^{p}\right)^{2} \mid}{\left(e_{i}^{p}\right)^{2}}}\right)
$$

Where $p$ denotes the $p$ th personnel capability, $\alpha_{i}^{p}$ is the weight of the $p$ th personnel capability for task $i, g_{i j}^{p}$ is the actual level of the $p$ th personnel capability of technical team $j$ for task $i, e_{i}^{p}$ is the required level of the $p$ th personnel capability for task $i$. In formula (1), If $g_{i j}^{p}>e_{i}^{p}$, take "+"; else take "_.".

The matching degree $T R_{i j}$ between task $i$ and team $j$ at the dimension of resource is defined as follows:

$$
T R_{i j}=\sum_{r=1}^{4} \beta_{i}^{r}\left(1 \pm \sqrt{\frac{\left(g_{i j}^{r}\right)^{2}-\left(e_{i}^{r}\right)^{2} \mid}{\left(e_{i}^{r}\right)^{2}}}\right)
$$

Where $r$ denotes the $r$ th resource, $\beta_{i}{ }^{r}$ is the weight of the $r$ th resource for task $i, g_{i j}{ }^{r}$ is the actual level of the $r$ th resource of technical team $j$ for task $i, e_{i}^{r}$ is the required level of the $r$ th resource for task $i$.

Furthermore, the matching degree $M D_{i j}$ between task $i$ and technical team $j$ is defined as:

$$
\left.\left.M D_{i j}=\omega_{i 1} \sum_{p=1}^{8} \alpha_{i}^{p}\left(1 \pm \sqrt{\frac{\left(g_{i j}^{p}\right)^{2}-\left(e_{i}^{p}\right)^{2} \mid}{\left(e_{i}^{p}\right)^{2}}}\right)\right)+\omega_{i 2} \sum_{r=1}^{4} \beta_{i}^{r}\left(1 \pm \sqrt{\frac{\left(g_{i j}^{r}\right)^{2}-\left(e_{i}^{r}\right)^{2} \mid}{\left(e_{i}^{r}\right)^{2}}}\right)\right)
$$

Where $w_{i 1}$ and $w_{i 2}$ are the weights of personnel capability and resource for task $i$, respectively.

Multi-objective optimization scheduling model. In collaborative innovation project, through rational resource selection and configuration according to project tasks' requirements, duration and cost optimal are achieved.

Parameters:

PT: the project duration; $C$ : the project cost; $T$ : the set of project tasks, $T=\left\{T_{1}, T_{2}, \ldots, T_{m}\right\}$;

$G$ : the set of technical teams, $G=\left\{G_{1}, G_{2}, \ldots, G_{n}\right\}, n$ is the number of technical team;

$s_{t i}:$ the start time of task $i, S=\left\{s_{t 1}, s_{t 2}, \ldots, s_{t i}, \ldots, s_{t m}, s_{t m+1}\right\}$, task $m+1$ is a virtual task; 
$M D_{i j}$ : the matching degree between task $i$ and technical team $j$;

$t_{N i}$ : the standard expected execution time of task $i$;

$\Delta t_{i}$ : the maximum shorten amplitude of execution time for task $i$;

$t_{i j}$ : the expected time of technical team $j$ to execute task $i$.

About scheduling objective trade-off, Chen et al proposed that there was linear relation between activity time reduction and cost increases, the time-cost trade-off problem can be transferred into a linear programming problem[10]. Thus, the optimization objective is as follows:

$$
\min f(x)=W_{1} * P T+W_{2} * C=W_{1} * S_{t_{m+1}}+W_{2} * C
$$

Constraints:

$$
\begin{aligned}
& x_{i j}= \begin{cases}1, & \text { team } \mathrm{j} \text { complete task } \mathrm{i} \\
0 & \text { else }\end{cases} \\
& e_{r \min }^{i} \leq e_{r}^{i} \leq e_{\text {max }}^{i} \\
& \sum_{j=1}^{n} x_{i j}=1 \\
& \sum_{i \in A_{t}} x_{i j}=1 \\
& S_{t q}=\max \min \left(S_{t i}+t_{i j}\right), T_{i} \in B_{(q)} \\
& t_{i j}=\left\{\begin{array}{c}
\frac{t_{N i}}{\sqrt{\mathrm{MD}_{\mathrm{ij}}}}, \quad \mathrm{MD}_{\mathrm{ij}}<\left(\frac{t_{N i}}{t_{N i}-\Delta t_{i}}\right)^{2} \\
t_{N i}-\Delta t_{i}, \quad \mathrm{MD}_{\mathrm{ij}} \geq\left(\frac{t_{N i}}{t_{N i}-\Delta t_{i}}\right)^{2}
\end{array}\right.
\end{aligned}
$$

Where $W_{1}$ and $W_{2}$ are the weights of project duration and cost respectively. Constraint(6) expresses resource constraint. Constraint(7) ensures that task $i$ just only be done by technical team $j$. Constraint(8) ensures that one team just only can perform one task at a period, $A_{t}$ is the collection of tasks carried out at time $t$. Constraint(9) denotes time constraint, $B(q)$ is the precedence activities set of task $q$. Equation(10) denotes the time will be taken for technical team $j$ to finish task $i$ while considering the matching degree $M D_{i j}$.

\section{The Improved Genetic Algorithm}

Based on single coding, multi-point crossover and simulated annealing operator, the genetic algorithm is improved to enhance the accuracy and reduce the complexity of computation.

The steps of the improved genetic algorithm are as follow:

Step 1: Coding.

Adopting decimal single coding, each gene locus represents the task code and the number on the gene locus represents the corresponding matching technical team.

Step 2: Selecting the initial population.

Randomly generate a certain number of individuals. Then, remove the repeated individuals and the individuals that do not meet the constraints, choose the best individual into the initial population and select a-1 individual from the remaining individuals randomly, compose initial population with number of a.

Step 3: Mutation operator.

In the process of mutation, single point mutation is used the first half of the gene, and multi-point mutation is adopted in the second part.

Step 4: Crossover operator.

Multi-point crossover is adopted. Cross points are selected randomly, and the amount of cross points is also selected randomly but greater than 1 .

Step 5: Selection operator.

The previous generation population, population after crossover and population after mutation, total $3 \mathrm{a}$ individuals are included in the selection set. Firstly, individuals that repeated and do not meet the constraints are removed. Then, the best individual of preceding generation population, crossover population and mutation population are retained respectively. For the remaining individuals, two 
individuals are selected randomly and one is chosen using simulated annealing operator with probability $\exp (-\Delta \mathrm{c} / \theta)$ to bring into the next generation, the another one is taken back. Repeat the above procedure until the amount of next generation reached number a, then turn into the step 3 .

Step 6: End, output the optimal.

When meet one of the conditions, the iteration is stopped:

(1) Fitness of the best individual and the group are no longer rising;

(2) The number of iterations reaches the preset number.

\section{Case Study}

The optimization model and algorithm were applied into a mobile phone design project. The relationship matrix of design task was shown in Figure 1. Totally 15 tasks were included in the project, the available technical team were G1, G2, .., G20, respectively.

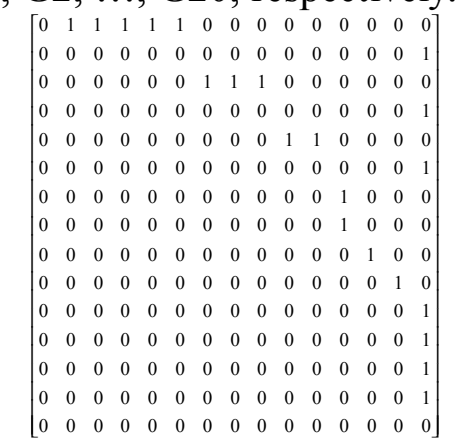

Fig.1 Task relationship matrix of design project

Standard execution time of the tasks and the maximum shorten times were shown in Table 2. The costs that technical team complete the tasks were in Table 3, and the matching degree between technical team and task were shown in table 4.

Table 2 Standard execution time and the maximum shorten time of design tasks

\begin{tabular}{|c|c|c|c|c|c|c|c|c|c|c|c|c|}
\hline \multicolumn{2}{|c|}{ Task } & \multicolumn{2}{|c|}{$\mathrm{T}_{1}$} & $\mathrm{~T}_{2}$ & $\mathrm{~T}_{3}$ & \multicolumn{2}{|c|}{$\mathrm{T}_{4}$} & $\mathrm{~T}_{5}$ & $\mathrm{~T}_{6}$ & & $\mathrm{~T}_{7}$ & $\mathrm{~T}_{8}$ [day] \\
\hline \multicolumn{2}{|c|}{$t_{N i}$} & \multicolumn{2}{|c|}{4} & 5 & 5 & \multicolumn{2}{|c|}{6} & 30 & 30 & & 25 & 7 \\
\hline \multirow{2}{*}{\multicolumn{2}{|c|}{$\begin{array}{l}\Delta t_{i} \\
\text { Task }\end{array}$}} & \multicolumn{2}{|c|}{1} & 2 & 3 & \multicolumn{2}{|c|}{2} & 2 & 3 & & 2 & 1 \\
\hline & & \multicolumn{2}{|c|}{$\mathrm{T}_{8}$} & $\mathrm{~T}_{9}$ & $\mathrm{~T}_{10}$ & \multicolumn{2}{|c|}{$\mathrm{T}_{11}$} & $\mathrm{~T}_{12}$ & $\mathrm{~T}_{13}$ & \multicolumn{2}{|r|}{$\mathrm{T}_{14}$} & $\mathrm{~T}_{15}$ \\
\hline & $t_{N i}$ & \multicolumn{2}{|c|}{7} & 5 & 15 & \multicolumn{2}{|c|}{5} & 1 & 1 & \multicolumn{2}{|r|}{1} & 4 \\
\hline \multicolumn{2}{|c|}{$\Delta t_{i}$} & 1 & & 2 & 3 & 2 & & 0.5 & 0.2 & & 0.5 & 2 \\
\hline & & \multicolumn{11}{|c|}{ Table 3 The cost that technical team complete the task } \\
\hline & $\overline{\mathrm{G}_{1}}$ & $\overline{\mathrm{G}_{2}}$ & $\mathrm{G}_{3}$ & $\mathrm{G}_{4}$ & $\mathrm{G}_{5}$ & $\overline{\mathrm{G}_{6}}$ & $\mathrm{G}_{7}$ & $\mathrm{G}_{8}$ & & $\mathrm{G}_{18}$ & $\mathrm{G}_{19}$ & $\mathrm{G}_{20}\left[10^{3}\right]$ \\
\hline $\mathrm{T}_{1}$ & 6 & 8 & 7 & 7 & 9 & 10 & 7 & 7 & & 8 & 6 & 9 \\
\hline $\mathrm{T}_{2}$ & 8 & 7 & 7 & 6 & 9 & 9 & 6 & 5 & & 6 & 5 & 8 \\
\hline $\mathrm{T}_{3}$ & 6 & 7 & 7 & 5 & 7 & 6 & 7 & 5 & & 7 & 5 & 5 \\
\hline $\mathrm{T}_{4}$ & 8 & 9 & 8 & 9 & 12 & 11 & 10 & 8 & & 9 & 7 & 12 \\
\hline $\mathrm{T}_{5}$ & 18 & 19 & 17 & 17 & 20 & 18 & 16 & 23 & & 21 & 16 & 24 \\
\hline $\mathrm{T}_{6}$ & 23 & 26 & 27 & 27 & 24 & 25 & 22 & 20 & & 23 & 20 & 26 \\
\hline$\Gamma$ & & & & & & & & & & & & \\
\hline $\mathrm{T}_{14}$ & 3 & 5 & 3 & 3 & 6 & 6 & 5 & 6 & & 3 & 3 & 7 \\
\hline $\mathrm{T}_{15}$ & 11 & 7 & 10 & 8 & 8 & 9 & 8 & 12 & $\cdots$ & 8 & 7 & 8 \\
\hline
\end{tabular}


Table 4 Matching degree between technical team and task

\begin{tabular}{ccccccccccccc}
\hline & $\mathrm{G}_{1}$ & $\mathrm{G}_{2}$ & $\mathrm{G}_{3}$ & $\mathrm{G}_{4}$ & $\mathrm{G}_{5}$ & $\mathrm{G}_{6}$ & $\mathrm{G}_{7}$ & $\mathrm{G}_{8}$ & $\ldots \ldots$ & $\mathrm{G}_{18}$ & $\mathrm{G}_{19}$ & $\mathrm{G}_{20}$ \\
\hline $\mathrm{T}_{1}$ & 1.974 & 0.501 & 1.361 & 1.617 & 1.149 & 1.448 & 0.470 & 1.471 & $\ldots \ldots$ & 1.831 & 0.534 & 2.325 \\
$\mathrm{~T}_{2}$ & 1.623 & 0.932 & 0.743 & 1.425 & 1.728 & 1.227 & 0.669 & 0.569 & $\ldots \ldots$ & 0.873 & 0.855 & 1.751 \\
$\mathrm{~T}_{3}$ & 1.063 & 1.755 & 0.913 & 0.778 & 0.787 & 1.566 & 0.524 & 0.823 & $\ldots \ldots$ & 1.845 & 1.498 & 1.376 \\
$\mathrm{~T}_{4}$ & 1.601 & 0.922 & 0.482 & 1.175 & 1.902 & 1.133 & 1.483 & 1.658 & $\ldots \ldots$ & 1.879 & 0.905 & 1.382 \\
$\mathrm{~T}_{5}$ & 1.478 & 1.230 & 0.609 & 1.701 & 1.904 & 1.623 & 1.175 & 1.246 & $\ldots \ldots$ & 1.997 & 1.370 & 1.494 \\
$\mathrm{~T}_{6}$ & 0.876 & 0.632 & 1.157 & 0.676 & 1.477 & 1.154 & 0.918 & 1.594 & $\ldots \ldots$ & 0.745 & 0.648 & 1.676 \\
$\Gamma$ & $\ldots \ldots$ & $\ldots \ldots$ & $\ldots \ldots$ & $\ldots \ldots$ & $\ldots \ldots$ & $\ldots \ldots$ & $\ldots \ldots$ & $\ldots \ldots$ & $\ldots \ldots$ & $\ldots \ldots$ & $\ldots \ldots$ & $\ldots \ldots$ \\
$\mathrm{T}_{14}$ & 1.054 & 1.280 & 1.591 & 1.241 & 0.768 & 1.641 & 0.563 & 0.414 & $\ldots \ldots$ & 1.509 & 1.531 & 1.292 \\
$\mathrm{~T}_{15}$ & 0.488 & 1.873 & 1.978 & 1.811 & 1.566 & 1.417 & 0.962 & 0.753 & $\ldots \ldots$ & 1.344 & 0.779 & 1.133 \\
\hline
\end{tabular}

The fitness function $\mathrm{f}(\mathrm{x})=0.6 * \mathrm{PT}+0.4 * \mathrm{C}$. Based on the data above, the procedures of the improved genetic algorithm were written by MATLAB, and the optimal program of task-team matching was got in 458th iteration and the result was shown in Table 5.

Table 5 Tasks - team matching program

\begin{tabular}{ccccccccc}
\hline Task & 1 & 2 & 3 & 4 & 5 & 6 & 7 & 8 \\
\hline technical team & 1 & 19 & 4 & 1 & 7 & 8 & 12 & 2 \\
\hline Task & 9 & 10 & 11 & 12 & 13 & 14 & 15 & \\
\hline technical team & 19 & 13 & 2 & 1 & 5 & 12 & 2 & \\
\hline
\end{tabular}

Under this matching program, the objective optimal value is 76.0222 , while duration is 46.7037 days and cost is $120 * 10^{3} \mathrm{RMB}$. The convergence curve of the objective function in the iterative process was shown in Figure 2. The running result by standard genetic algorithm was shown in Figure 3. The objective function value of the optimal was 76.4222. The iteration number of finding the optimal was greater than the improved algorithm. The result of the comparison displayed the advantage of the improved algorithm in finding the optimal and convergence speed.
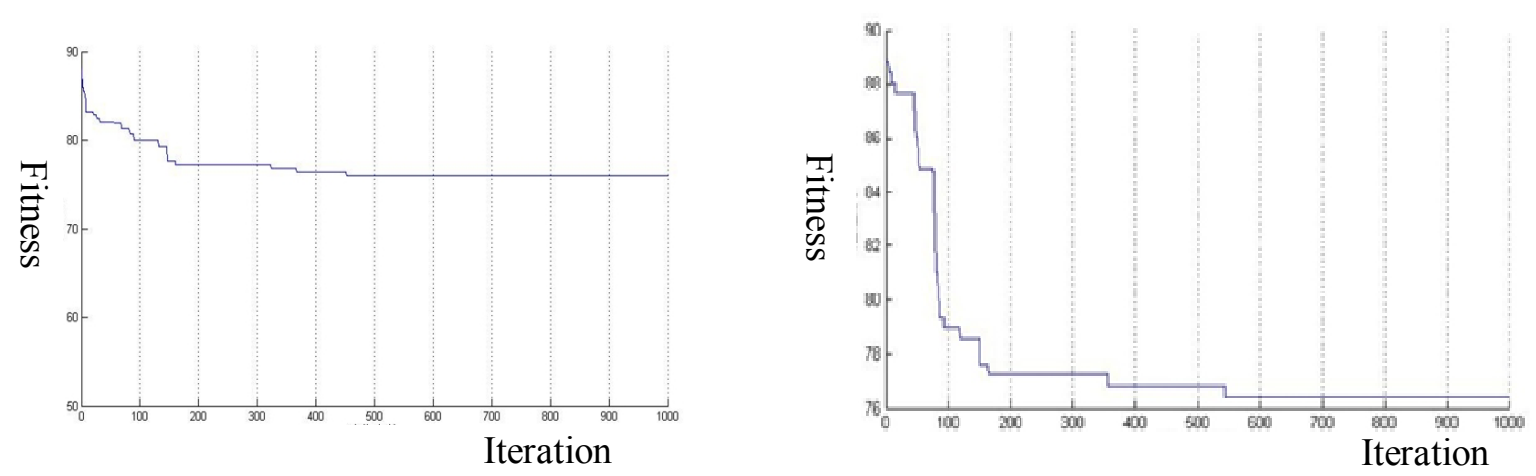

Fig. 2 Convergence curves of the objective function Fig.3 The running result of traditional GA The project task allocation and scheduling plan was shown in Figure 4. 


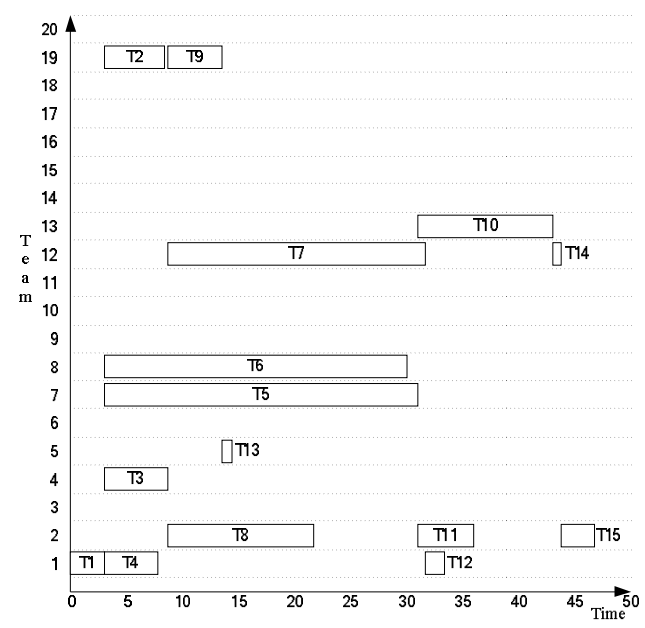

Fig.4 Project task allocation and timing chart

\section{Conclusions}

Resources allocation of collaborative innovation project has an important influence on innovation efficiency and cost. Thus, competence elements for CPD are presented. Then, the definition of matching degree is applied to describe the fitness between team and task. On the basis, a scheduling model for collaborative product design based on MD is established, and the improved genetic algorithm is adopted to search the optimal solution. Based on single-coding strategy, multi-point cross and combined with simulated annealing operator, the performance of the algorithm is improved. Finally, the feasibility and efficiency of the proposed model and algorithm are verified. In the future, we shall focus on how to improve innovative efficiency from innovation agent interaction.

\section{Acknowledgements}

This research is supported by Natural Science Foundation of Hebei province (No.G2013502228), the Fundamental Research Funds for the Central Universities (No.2014 MS120) and the Science Foundation of Hebei Finance University (No.JY201506).

\section{References}

[1] Y. H. Lee, C. T. Chang, D. S. Wong, S. S. Jang, Chemical Engineering Research and Design. 89 (2011) 291-300.

[2] T. R. Wang, S. S. Guo, B. R. Sarker, Y. B Li, P Advanced Engineering Informatics. 26 (2012) 280-291.

[3] M. E. Bruni, P. Beraldi, F. Guerriero, Computers \& Operations Research. 38 (2011) 1305-1318.

[4] A. Tahooneh, K. Ziarati, Using artificial bee colony to solve stochastic resource constrained project scheduling problem, Advances in Swarm Intelligence, Proceedings of the Second International Conference. 2011, 293-302.

[5] H. Pang, Z. D. Fang, H. Guo, Y. Zhao, Systems Engineering and Electronics, 30 (2008) 1899-1903(In Chinese).

[6] Y. Zhao, Q. Song, Advances in Information Sciences and Service Sciences. 4 (2012) 109-117.

[7] Y. H. Wu, X. S. Qin, Industrial Engineering Journal. 14 (2011) 80-84(In Chinese).

[8] S. Zhang, Y. Z. Li, Computer Modeling and New Technologies. 18 (2014) 25-30.

[9] B. Du, J. F. Li, Science Research Management. 33 (2012) 40-48 (In Chinese).

[10] S. P. Chen, M. J. Tsai, European Journal of operational research. 212 (2011) 386-397. 